\title{
Variations in process and outcomes of diabetes care by socio-economic status in Salford, UK
}

\author{
R. Edwards ${ }^{1}$, J. A. Burns ${ }^{2}$, P. McElduff ${ }^{1}$, R. J. Young ${ }^{2}$, J. P. New ${ }^{2}$ \\ ${ }^{1}$ Evidence for Population Health Unit, School of Epidemiology and Health Sciences, The Medical School, \\ The University of Manchester, Manchester, United Kingdom \\ 2 Department of Diabetes, Hope Hospital, Salford, UK
}

\section{Abstract}

Aims/hypothesis. Our aim was to investigate trends in provision and outcomes of care by socio-economic status among patients with diabetes in Salford, United Kingdom.

Methods. Salford is a deprived urban area in North West England. Data for people with diabetes who were younger than 20 years of age (4034 patients in the year 1993 and 5671 by the year 2000) were extracted from the Diabetes Information System. Agestandardised means, proportions and attainment of targets were calculated for: processes of care indicators, intermediate outcomes and prescribing of preventive drug treatments in 1993 to 1994 and in 2000 to 2001 by quintiles of Townsend deprivation score. We did comparisons of means and proportions using ageadjusted linear regression and of trends using generalised estimating equations. Rate ratios for first microvascular and first macrovascular complication were estimated from proportional hazards models.

Results. Marked improvements occurred in all indicators. For patients managed in primary care, blood pressure and cholesterol measurement increased from $53 \%$ to $64 \%(p<0.001)$ and $27 \%$ to $61 \%(p<0.001)$ respectively; whilst mean systolic blood pressure decreased from 147 to $140 \mathrm{mmHg}(p<0.001)$ and cholesterol concentrations from 6.0 to $5.1 \mathrm{mmol} / \mathrm{l}(p<0.001)$. Mean $\mathrm{HbA}_{1 \mathrm{c}}$ increased from 7.8 to $8.1 \%(p<0.001)$. Prescribing of aspirin, anti-hypertensive and lipidlowering drugs increased greatly. Trends varied little by socio-economic status. Patients from more affluent areas generally received more frequent clinical monitoring and preventive treatments, and had a lower BMI (29.5 vs $\left.30.2 \mathrm{~kg} / \mathrm{m}^{2} ; p=0.009\right)$ and $\mathrm{HbA}_{1 \mathrm{c}}(7.8 \mathrm{vs}$ $8.2 \% p=0.006)$, though risks of first microvascular or macrovascular complications were similar.

Conclusion/interpretation. Improvements in process and outcomes of care are possible for patients from all socio-economic groups. Socio-economic deprivation does not preclude high quality diabetes care. [Diabetologia (2003) 46:750-759]

Keywords Diabetes mellitus, socio-economic status, quality of care, structured care, information systems.
Received: 19 November 2002 / Revised: 10 February 2003 Published online: 23 May 2003

(C) Springer-Verlag 2003

Corresponding author: Dr. R. Edwards, Evidence for Population Health Unit, School of Epidemiology and Health Sciences, The Medical School, The University of Manchester, Oxford Road, Manchester, M13 9PT, United Kingdom

E-mail: Richard.Edwards@man.ac.uk

Abbreviations: DIS, Diabetes information system; SES, socioeconomic status.
Most studies suggest that the prevalence of risk factors for diabetic complications, intermediate outcomes of diabetes care, and diabetic complication rates vary inversely with socio-economic status (SES). For example, risk factors for cardio-vascular diseases, particularly cigarette smoking, are more prevalent among people with diabetes with lower SES in community and hospital-based studies [1, 2, 3, 4, 5]. Patients of lower SES have an increased incidence of retinopathy and nephropathy $[1,2,4,5]$.

All cause mortality is higher in people with diabetes of lower SES [6] or from more deprived communi- 
ties [7]. The Whitehall study and the London cohort of the World Health Organization (WHO) study of vascular disease in people with diabetes showed clear socio-economic gradients in the prevalence of heart disease. All cause age-adjusted mortality, and mortality from ischaemic heart disease and all cardio-vascular diseases were higher in patients with diabetes with lower SES. These differences persisted after adjustment for smoking status and blood pressure, though in some cases the trend across SES groups became statistically non-significant [2]. In contrast, a large Finnish study did not find mortality differentials by social class [8].

There is clear evidence that improved glycaemic control reduces the development and progression of microvascular and macrovascular complications of diabetes [9]. One explanation for the poorer outcome in diabetes with increasing deprivation is suggested by the association of poorer glycaemic control with lower SES, which has been shown in some, but not all, studies. For example, studies from the United Kingdom $[4,10]$ as well as a large population-based study from Germany [5] have shown that for people with diabetes being treated with insulin, $\mathrm{HbA}_{1 \mathrm{c}}$ values were higher among patients from more deprived areas. Another study from the United Kingdom found no association between SES and $\mathrm{HbA}_{1 \mathrm{c}}$ values [11].

Variations in health by socio-economic status among people with diabetes could reflect the direct effects of deprivation on health or could result indirectly from the effects of adverse health behaviours linked to lower SES. Another possible explanation is the 'inverse care law' [12] whereby access to and take up of services is less, and the quality of care provided poorer, for patients with the greatest need.

The data about variations in access and take up of care, and the quality of care by socio-economic status for people with diabetes mostly supports the notion of the inverse care law. For example, a cross-sectional study found that fewer practices in more deprived urban areas provided diabetes management programmes than practices in more affluent rural and sub-urban areas [13]. In another cross-sectional study of 260 practices in 1996, practices serving more deprived populations were less likely to have a glucometer or access to chiropodists and dieticians. However, the practice population SES score (Townsend or Jarman) did not predict the likelihood of having a mini-clinic or recall system for people with diabetes [14]. If access to and use of diabetic services is poorer among lower SES groups, possible explanations include poorer knowledge about diabetes and less participation in educational programmes [5].

Studies investigating the process of care for people with diabetes have often found variations in relation to SES of patients and of their practices. In the EURODIAB study, a cross-sectional study of patients with Type 1 diabetes from 31 European clinics, hyper- tensive treatment did not vary by educational level. However, a markedly lower proportion of people with diabetes who had completed only primary education had had an $\mathrm{HbA}_{1 \mathrm{c}}$ measurement in the previous two years [1]. In a subset of 149 practices from 260 in a study, which had taken part in multi-practice diabetes audits, patients from practices serving more deprived populations were less likely to have had $\mathrm{HbA}_{1 \mathrm{c}}$, BP, fundi and their feet checked [15]. Findings from the Teesside group's hospital-based study suggested that patients from more deprived areas were less likely to be receiving insulin treatment despite poorer glycaemic control $[4,10]$. However, this was potentially confounded by an increased prevalence of Type 2 diabetes among the people with diabetes from more deprived groups [4]. Another study found that deprivation scores did not predict the quality of care for diabetes, as measured by a complex score encompassing a wide range of process of care measures and intermediate outcomes [16].

An important limitation of most previous studies describing the association of process measures, intermediate outcomes and complication rates with SES, has been their cross-sectional nature. Trends are therefore unclear, particularly whether variations by socio-economic status are increasing or decreasing. The aim of this study was to use a Diabetes Information System (DIS) to investigate trends in process of care measures, prescribing of preventive drug treatments, intermediate outcomes, and complication rates by socio-economic status among patients with diabetes in a mostly deprived urban population. We hypothesise that process of care measures, intermediate outcomes, and complication rates will be better, and improvement between 1993 and 2001 will be greater, among patients with diabetes from more affluent areas.

\section{Subjects and methods}

Setting. Salford is an urban district in Greater Manchester, United Kingdom, with a population of about 225000. Approximately half of the electoral wards are moderately or severely deprived, and Salford is ranked 29th out of 354 English Local Authorities for income deprivation. The high level of deprivation is reflected in poor health experience on a range of indicators compared to regional and national averages-including reduced life expectancy, higher overall and cause specific mortality from cancers and cardio-vascular diseases, and higher rates of teenage pregnancies. Less than $5 \%$ of the population is of non-European ethnicity [17].

The Salford Diabetes Information System. A population based Diabetes Information System was established in the Salford district in 1992 to support a guideline-based integrated diabetes care programme, introduced 4 years previously. The programme aimed to improve the quality of diabetes care by prompting and supporting diabetes care providers throughout Salford. Within Salford, all patients diagnosed with diabetes, 
both in primary and secondary care, are registered on the DIS as this is used to facilitate structured care.

The DIS has been described in detail previously [17]. Briefly, the DIS represents a continuously updated diabetes health care record for all patients registered as having diabetes. The DIS prompts structured diabetes care using an automated recall system. Key processes of diabetes care (e.g. weight, BP, glycaemic control measurement; retinal screening; and foot examination) are prompted at an annual structured preventative care review using a standard clinic proforma.

The DIS records are based on the United Kingdom Diabetes dataset [18]. All information in the DIS records derives from clinical measurements and observations. These records are updated and verified during the annual review. Information is aggregated within the DIS from many sources including the biochemistry department, clinical optometrists, community podiatry, primary and secondary care. Outcome data, such as amputations, myocardial infarction and retinal laser photocoagulation are validated yearly, by linking the details recorded on DIS with outpatient and inpatient episodes coded through Hospital Episode Statistics [20]. Deaths data are captured by linkage with the national strategic tracing service.

The DIS provides annual feedback to all users relating to their provision of care. This feedback is linked to an ongoing programme of multidisciplinary professional education, covering the main aspects of diabetes care over a two-year cycle. Other district-wide changes in the provision of care during the study period included: revisions to district guidelines; the development of nurse-led clinics for hypertension and hypercholesterolaemia; and offering all newly-diagnosed patients the opportunity to attend education sessions.

All practices in Salford participate in a shared-care program and provide data for the DIS. All patients are seen in primary care with some patients also attending the hospital diabetes clinic ('shared-care' patients). 'Primary care' patients are managed solely in primary care.

Patients with diabetes are registered with the DIS by the clinician or other responsible health professionals.

Study population. Patients with diabetes on the DIS were included in the analysis if they were 20 years of age or older, alive, and were living within Salford (as defined by their postcode).

Data items and definitions. Postcodes for each patient on the diabetes register were mapped to enumeration districts, and assigned Townsend scores calculated in relation to all of England and Wales using data from the 1991 census. Townsend scores are a composite area-based indicator of social deprivation based on four indicators included in the United Kingdom decennial census [21]. A higher Townsend score indicates increasing deprivation.

Patients were classified as having Type 1 diabetes mellitus if they were diagnosed as having diabetes at an age younger than 35 years and treated with insulin within the first year. All other patients were assumed to have Type 2 diabetes mellitus. The main process and intermediate outcome measures used were measurement and level of $\mathrm{BP}$, serum cholesterol, $\mathrm{HbA}_{1 \mathrm{c}}$ and BMI for each year. Targets for glycaemic control, cholesterol and blood pressure were defined in the local guidelines in 1994 as: $\mathrm{HbA}_{1 \mathrm{c}}$ less than $9 \%$ (normal range <6.0\%; measured by the Cobas Integra 700 analyser using a DCCT standardized assay); total cholesterol less than $5.5 \mathrm{mmol} / \mathrm{l}$, systolic BP less than $160 \mathrm{mmHg}$ and diastolic BP less than $90 \mathrm{mmHg}$. In 1998 the targets were changed to BP less than $140 / 80 \mathrm{mmHg}$ in keeping with the 1999 British Hypertension Society guidelines [19], glycaemic control $\left(\mathrm{HbA}_{1 \mathrm{c}} \leq 7.5 \%\right)$, and cholesterol con- centrations of less than $5.0 \mathrm{mmol} / \mathrm{l}$. The 1998 target levels were used in all analyses.

Patients were categorised as receiving aspirin, anti-hypertensive or lipid-lowering drugs according to the DIS record. Patients were classified as having microvascular disease if the DIS indicated the presence of any form of diabetic retinopathy or neuropathy; and macrovascular disease if they had a stroke, transient ischaemic attack, myocardial infarction, angina, or peripheral vascular disease.

Statistical analysis. Subjects were categorised for analysis into quintiles of deprivation based on the Townsend score of the enumeration district in which they live. Data are presented for: $\mathrm{BMI}, \mathrm{BP}, \mathrm{HbA}_{1 \mathrm{c}}$, total serum cholesterol; and treatment with aspirin, lipid-lowering and anti-hypertensive drugs. For each physiological variable, the proportion of patients with a measurement recorded, the mean level, and the proportion attaining target levels were calculated by averaging separately the results for the years 1993 and 1994, and for 2000 and 2001stratified by type of care provision. The proportion receiving treatment with aspirin, BP or lipid lowering agents were also calculated. All values were age-standardised by the direct method using the age distribution of all the patients older than 20 years of age who lived in Salford and were registered on the DIS system in 1993.

Tests for trend across the five quintiles of deprivation were calculated using regression models. For these and other analyses we have presented absolute $p$ values, however, wherever we have reported statistically significant findings in the text, we have used a $p$ value of less than 0.05 as a cut-off point.We also estimated the average annual change in the above variables between 1993 and 2001 using all available data. The data consist of repeated measurements on a changing population and therefore the usual regression techniques could produce biased estimates for the standard errors of regression coefficients. To ensure robust estimates we used generalised estimating equations with an identity link and a normally distributed error term [23]. The coefficient of the year variable from these models is an estimate of the average annual change in the mean level of continuous variables or the average annual absolute change in the proportion for dichotomous variables. All analyses were carried out using Stata version 7.0 statistical software.

Rate ratios were calculated separately for first microvascular and first macrovascular complication between 1993 and 2001 for each quintile of deprivation using proportional hazards models. The rate ratios were calculated after adjusting for age, sex, type of diabetes and type of care. The models only included those who had no known history of a microvascular or macrovascular complication at the time of registration with the DIS.

Analyses were carried out in accordance with the Declaration of Helsinki. All patients gave their verbal consent to the storage and use of their data to monitor and improve clinical care. Data used for the analysis is made fully anonymous with all individual patient identifiers removed and only aggregated results were reported to protect patient confidentiality.

\section{Results}

At the beginning of 1993 there were 4034 patients (653 with Type 1 diabetes mellitus) aged 20 years or older, alive and included on the DIS. By the year 2000 there were 5671 (585 with Type 1 diabetes) patients. Townsend scores were assigned to $97.3 \%$ in the year 
Table 1. Comparison of patient characteristics in 2000— by quintile of Townsend score ${ }^{\mathrm{a}}$

Variable of interest
Quintile of Townsend deprivation score ${ }^{b}$

123

$(n=1124) \quad(n=1076) \quad(n=1087)$

64.0

63.5

63.5
47.0

65.8

54.9

62.1

53.9

12.3
53.8

10.2
Age (mean)

Type 1diabetes mellitus

Type 2 diabetes mellitus

Men (\%)

Type 1 diabetes mellitus

Type 2 diabetes mellitus

$\%$ of Type 1 diabetes mellitus among all diabetic patients

49.7

65.9

55.3

54.1

55.4

11.8

Treatment For Type 2 diabetes mellitus $(\%)^{\mathrm{d}}$

Oral hypoglycaemic tablets only $\quad 72.7$

Insulin only

Insulin and oral hypoglycaemic tablets

Mean duration of diabetes (years)

Type 1 diabetes mellitus

Type 2 diabetes mellitus

$\%$ Shared care

Type 1 diabetes mellitus

Type 2 diabetes mellitus
12.6

14.7

8.7

19.6

7.3

42.8

85.7

37.0

$(n=1087) \quad(n=1072)$

5

$(n=1076)$

63.8

48.5

65.3

49.8

49.5

49.9

8.6

49.2

9.0

$p$ value for

test for

linear trend ${ }^{c}$

.

\footnotetext{
a The Townsend quintiles in this and subsequent tables and figures represent scores of: 1 is $\leq 1.48 ; 2$ is $>-1.48$ and $\leq 1.26 ; 3$ is $>1.26$ and $\leq 3.32 ; 4$ is $>3.32$ and $\leq 5.60 ; 5$ is $>5.60$. A higher number represents a more deprived area

b Subjects for whom a Townsend score not be assigned have been excluded $(n=236)$
}

1993 and $95.3 \%$ in the year 2000. Patients without Townsend scores were excluded from the subsequent analysis ( $n=236$ in the year 2000).

In $2000,71 \%$ of the patients on the DIS came from areas that had a Townsend score greater than the median for England. Table 1 shows descriptive data for the study population in the year 2000 by type of diabetes and quintiles of deprivation. When analysed by quintile of deprivation, there were no statistically significant differences in age distribution. However, the proportion of patients with Type 2 diabetes who were men was lowest in more deprived quintiles. The proportion of patients with Type 1 diabetes was lower in patients living in the most deprived areas. Patients with diabetes from the more deprived areas had a shorter mean duration of diabetes. There was evidence of poorer access and delivery of care for patients with diabetes from more deprived areas. These patients were less likely to be receiving shared care, and patients with Type 2 diabetes who were on treatment were less likely to be receiving insulin treatment (patients with Type 2 diabetes and no recorded diabetes treatment on the DIS were excluded from this analysis).

Table 2 and Table 3 show cross-sectional and trend data between 1993 to 1994 and 2000 to 2001 for pro- cess of care and the intermediate outcome measures for patients cared for in primary care exclusively and through shared care. In both groups, process of care indicators improved steadily throughout the study period. Measurement was highest for $\mathrm{HbA}_{1 \mathrm{c}}$ measurement in 2000 to 2001. There were large decreases in systolic and diastolic BP (diastolic BP decreased from 82.2 to $78.8 \mathrm{mmHg}$ in primary care patients $p<0.001$ and from 82.0 to $73.0 \mathrm{mmHg}$ in shared care patients, $p<0.001)$ and cholesterol concentrations, and concomitant improvements in attainment of target levels of control for these indicators. However, there were small increases in $\mathrm{HbA}_{1 \mathrm{c}}$ and $\mathrm{BMI}$, and worsening attainment of their target levels during the study period.

The most affluent patients tended to have the highest and the most deprived patients to have the lowest rate of measurement for each of these variables in primary care and shared care patients. By 2000 to 2001, there were no statistically significant trends in BP and cholesterol concentrations or target attainment by SES. However, $\mathrm{HbA}_{1 \mathrm{c}}$ and BMI were lower in the least deprived group, particularly among primary care patients for the former and shared care for the latter. The average annual absolute change in measurement, mean, and attainment of targets was generally similar 
R. Edwards et al.: Variations in process and outcomes of diabetes care by socio-economic status in Salford, UK

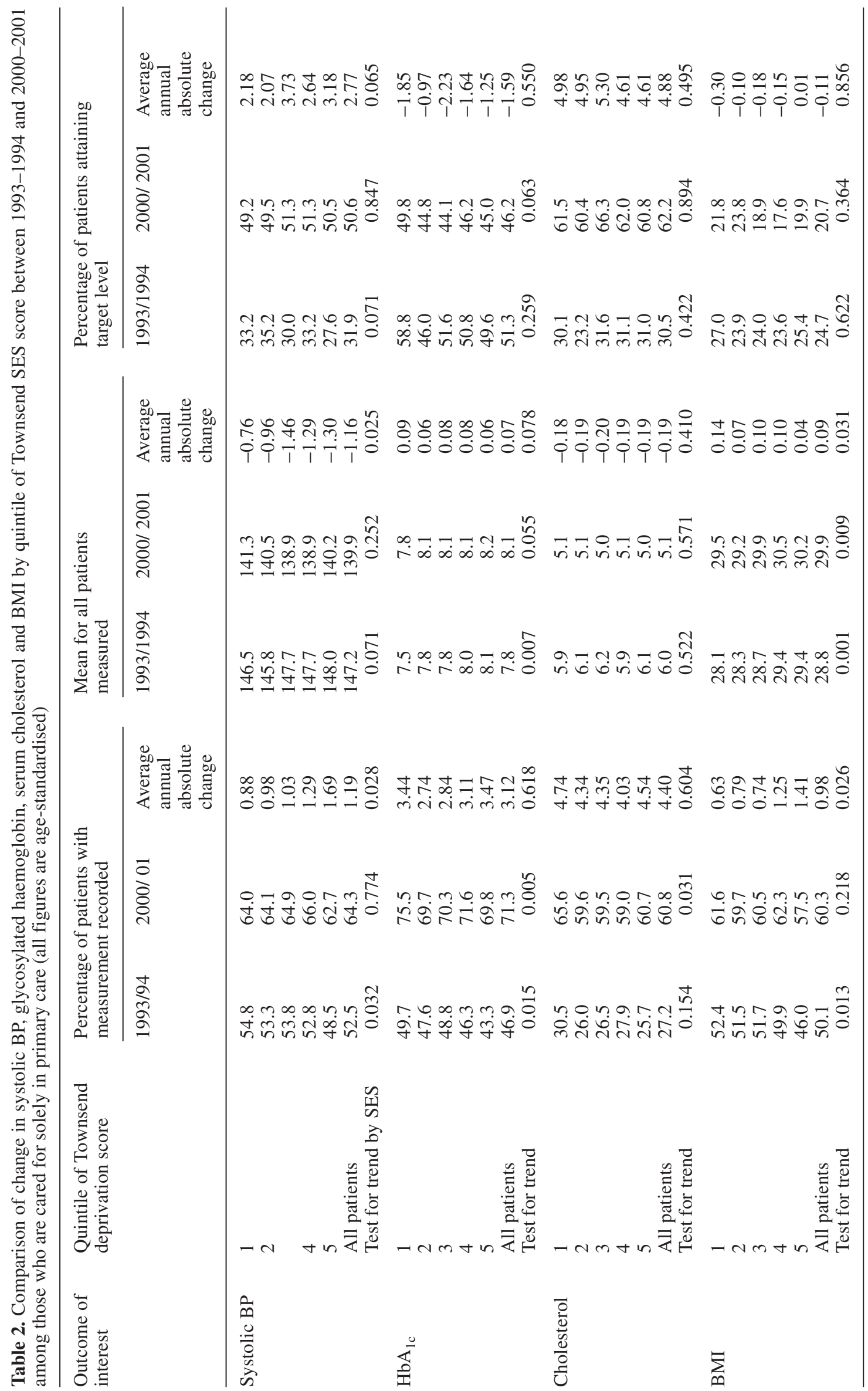




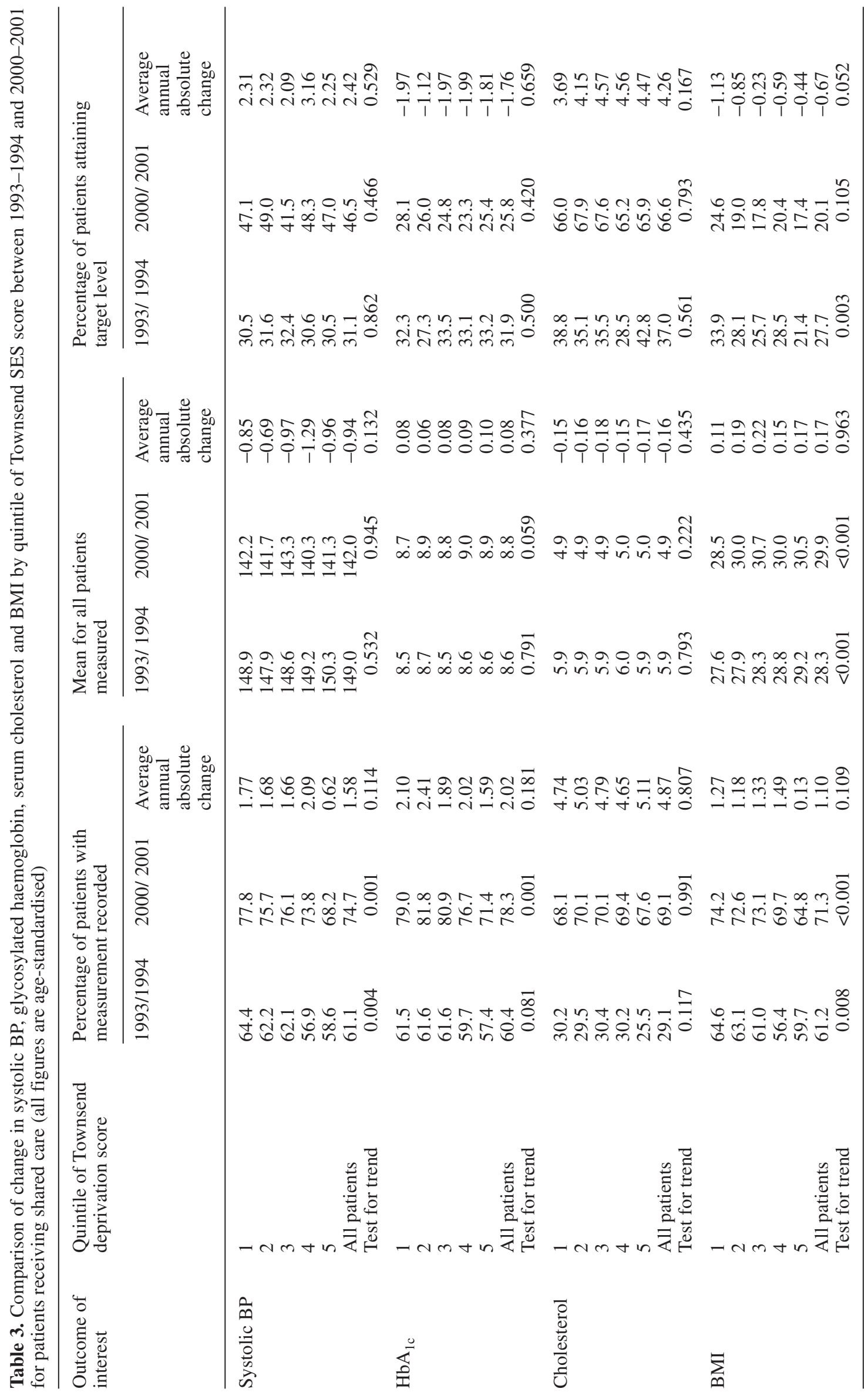




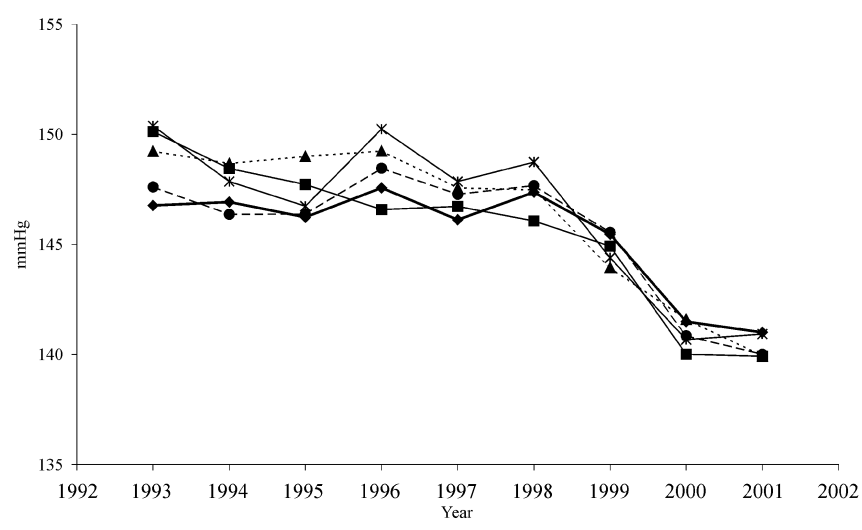

Fig. 1. Trends in the mean of systolic blood pressure among patients who had their blood pressure recorded by quintile of Townsend score

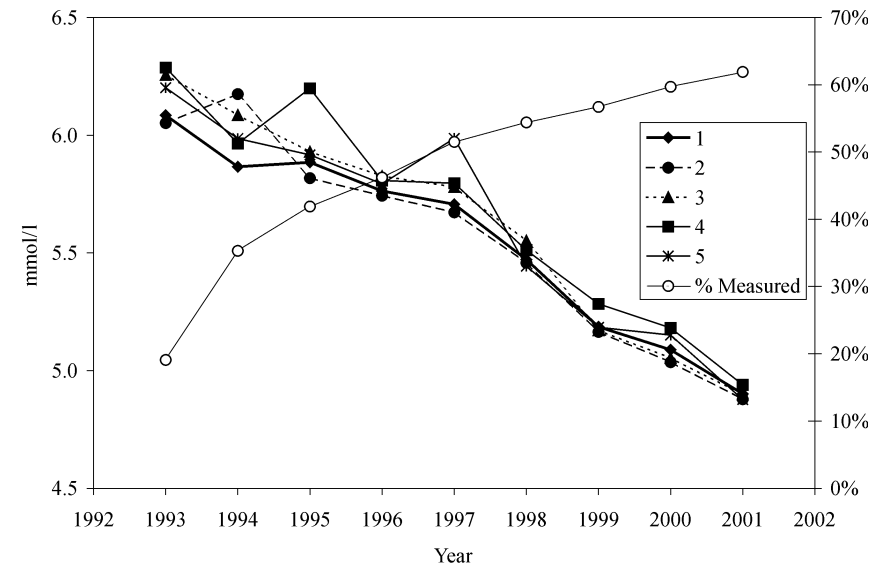

Fig. 2. Trends in the mean of serum cholesterol among patients who had their cholesterol concentrations recorded by quintile of Townsend score and trends in the percentage of patients who had their level of serum cholesterol recorded

Table 4. Trends in treatment by quintile of SES score 1993-2001

\begin{tabular}{|c|c|c|c|c|}
\hline \multirow[t]{2}{*}{ Treatment } & \multirow[t]{2}{*}{ Quintile of Townsend deprivation score } & \multicolumn{3}{|c|}{ Percentage of patients receiving treatment } \\
\hline & & 1993 & 2001 & $\begin{array}{l}\text { Average annual } \\
\text { absolute increase }\end{array}$ \\
\hline Anti-hypertensive & $\begin{array}{l}1 \\
2 \\
3 \\
4 \\
5 \\
\text { All patients } \\
p \text { value for test for linear trend }\end{array}$ & $\begin{array}{l}18.9 \\
20.8 \\
20.1 \\
19.5 \\
19.9 \\
19.8 \\
0.810\end{array}$ & $\begin{array}{l}50.5 \\
49.5 \\
51.5 \\
51.6 \\
52.3 \\
51.1 \\
0.071\end{array}$ & $\begin{array}{l}5.73 \\
5.44 \\
5.74 \\
5.42 \\
5.81 \\
5.63 \\
0.378\end{array}$ \\
\hline Lipid-lowering & $\begin{array}{l}1 \\
2 \\
3 \\
4 \\
5 \\
\text { All patients } \\
p \text { value for test for linear trend }\end{array}$ & $\begin{array}{l}3.4 \\
2.9 \\
3.5 \\
3.1 \\
4.1 \\
3.4 \\
0.217\end{array}$ & $\begin{array}{l}25.5 \\
29.6 \\
27.9 \\
27.5 \\
27.0 \\
27.5 \\
0.911\end{array}$ & $\begin{array}{l}3.59 \\
4.22 \\
3.85 \\
3.95 \\
3.67 \\
3.85 \\
0.411\end{array}$ \\
\hline Aspirin & $\begin{array}{l}1 \\
2 \\
3 \\
4 \\
5 \\
\text { All patients } \\
p \text { value for test for linear trend }\end{array}$ & $\begin{array}{l}0.8 \\
1.2 \\
1.5 \\
1.0 \\
0.3 \\
0.9 \\
0.403\end{array}$ & $\begin{array}{l}24.9 \\
25.2 \\
26.7 \\
25.2 \\
25.4 \\
25.5 \\
0.194\end{array}$ & $\begin{array}{l}3.84 \\
3.70 \\
3.65 \\
3.68 \\
3.73 \\
3.72 \\
0.123\end{array}$ \\
\hline
\end{tabular}

for all indicators across the SES groups for shared care and primary care patients.

The trends in systolic BP and cholesterol by SES are shown (Fig. 1, Fig. 2). Systolic BP decreased greatly, particularly after the year 1998 (Fig. 1). The rate of decrease was similar across all quintiles of deprivation. The mean cholesterol concentrations also decreased greatly and at a similar rate for all quintiles of deprivation (Fig. 2). Unlike the trends for mean $\mathrm{BP}$, the graph for cholesterol concentrations shows a steady decrease over the entire follow-up period.

The proportion of patients treated with anti-hypertensive or lipid-lowering treatment, or with aspirin are shown (Table 4). For all three treatments there were large increases in the proportion treated. There were no notable differences in the proportion treated at baseline, currently, or in the rate of increase in treatment by SES quintile. Whilst anti-hypertensive treatment increased steadily throughout the study, treat- 
ment with aspirin and lipid-lowering drugs remained uncommon until the year 1997, after which there was a steady increase (Fig. 3).

There were no statistically significant differences in microvascular and macrovascular complication rates and rate ratios for the development of complications during the study period by quintiles of deprivation (Table 5, Table 6).

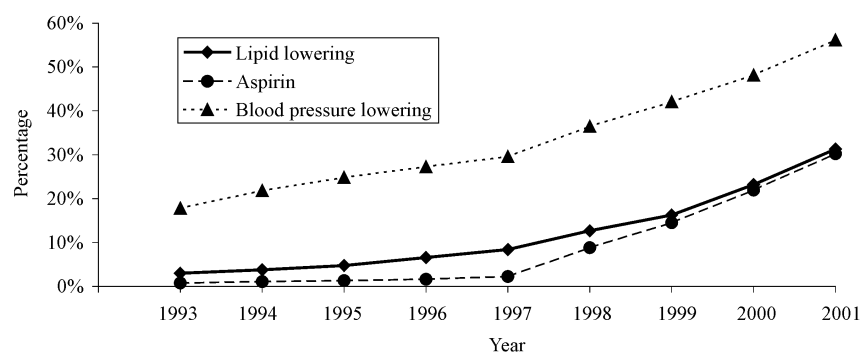

Fig. 3. Trends in the percentage of patients who were treated with aspirin, and antihypertensive medication or lipid lowering drugs

\section{Discussion}

This study has used data available from a comprehensive DIS to investigate cross-sectionally and over time the relation between SES and diabetes process of care and intermediate and final outcomes. The study, in a large urban population of patients with diabetes, shows that clinically important improvements in the process and outcomes of diabetes care occurred and that these changes were similar irrespective of socioeconomic status.

This paper builds on previous work showing improvements in the process and outcomes of care in Salford following implementation of the DIS. It augments this work by adding additional measures, i.e. more years of data and, most importantly, an investigation of variations by SES. Particular strengths of the study include its setting in everyday routine clinical practice, the large numbers of patients included, the extensive dataset, and the availability of longitudinal data over a 9 year period.

Among the striking findings are the marked improvement in the measurement and recording of a va-

Table 5. Macro vascular complications - by quintile of Townsend score

\begin{tabular}{|c|c|c|c|}
\hline \multirow[t]{2}{*}{ Quintile of Townsend deprivation score } & \multicolumn{2}{|c|}{ Patients with macrovascular complications (\%) } & \multirow{2}{*}{$\begin{array}{l}\text { Rate ratio for first } \\
\text { macrovascular } \\
\text { complication 1993-2001 } \\
(95 \% \text { CI })^{\mathrm{a}}\end{array}$} \\
\hline & $\begin{array}{l}\text { Prevalence at baseline } \\
\text { (start of 1993) }\end{array}$ & $\begin{array}{l}\text { Incidence of first } \\
\text { macrovascular } \\
\text { complications during } \\
2000\end{array}$ & \\
\hline 1 & 16.4 & 3.1 & 1.00 \\
\hline 4 & 18.2 & 5.0 & $1.03(0.69,1.52)$ \\
\hline 5 & 16.8 & 3.8 & $1.07(0.73,1.58)$ \\
\hline All patients & 16.7 & 4.0 & \\
\hline$p$ value for test for linear trend a & 0.166 & 0.261 & 0.341 \\
\hline
\end{tabular}

a Adjusted for age, sex, type of diabetes and provider of care

Table 6. Microvascular complications-by quintile of Townsend score

\begin{tabular}{|c|c|c|c|}
\hline \multirow[t]{2}{*}{ Quintile of Townsend deprivation score } & \multicolumn{2}{|c|}{ Patients with microvascular complications $(\%)$} & \multirow{2}{*}{$\begin{array}{l}\text { Rate ratio for first } \\
\text { microvascular } \\
\text { complication 1993-2001 } \\
(95 \% \text { CI })^{\mathrm{a}}\end{array}$} \\
\hline & $\begin{array}{l}\text { Prevalence at baseline } \\
\text { (start of 1993) }\end{array}$ & $\begin{array}{l}\text { Incidence of first } \\
\text { microvascular } \\
\text { complications during } \\
2000\end{array}$ & \\
\hline 1 & 11.2 & 4.4 & 1.00 \\
\hline 4 & 13.4 & 2.9 & $1.42(0.80,2.51)$ \\
\hline 5 & 12.8 & 2.9 & $1.23(0.68,2.21)$ \\
\hline All patients & 13.2 & 3.8 & \\
\hline$p$ value for test for linear trend ${ }^{a}$ & 0.148 & 0.029 & 0.322 \\
\hline
\end{tabular}

a Adjusted for age, sex, type of diabetes and provider of care 
riety of key intermediate outcomes during the study period. There were also steady increases in treatment with anti-hypertensive and lipid-lowering treatments and aspirin, prompted by revisions in the district guidelines as new evidence was introduced into clinical practice. There were contrasting findings on changes in the quality of care as measured by four intermediate outcome measures. Blood pressure and lipid concentrations decreased substantially. However, glycaemic control and BMI deteriorated during the study period, though in clinical practice the differences were small, with the $\mathrm{HbA}^{1 \mathrm{c}}$ increasing by 0.2 and $0.3 \%$. The generally positive findings of improvements in the process and outcomes of diabetic care, suggest that the DIS mechanism is an effective model for ongoing monitoring and improvement of diabetes care.

The decrease in systolic BP was most marked from the year 1999 to 2000, coinciding with the period during which the benefits of tighter BP control in patients with diabetes became clearer with the publication of key findings from the United Kingdom Prospective Diabetes Study (UKPDS) [24] and widespread dissemination of recommendations for tighter BP control advocated in the 1999 British Hypertension guidelines [22]. Reducing mean BP to $140 / 80 \mathrm{mmHg}$, which is slightly better than that in the UKPDS intensive BP treatment group is a considerable achievement. Cholesterol concentrations decreased steadily throughout the study period. This could be interpreted as showing the effect of successful management or to be simply an effect of including a lower risk group, as screening was extended to a greater proportion of patients with diabetes. However, the greatest increase in the proportion measured occurred in 1993 to 1997, whereas the decrease in cholesterol concentrations was greatest in 1997 to 2001 during the period when prescribing of lipid-lowering drugs was increasing most rapidly. Therefore, increased measurement is not likely to be the only explanation for the rapidly decreasing mean cholesterol concentrations.

Analysis by SES did show some stratification by level of deprivation. However, this was largely confined to the most deprived patients with diabetes being slightly less likely to be receiving shared care, insulin treatment (Type 2 diabetes mellitus) and to have measurements of $\mathrm{BP}$, cholesterol, $\mathrm{HbA}_{1 \mathrm{c}}$ and $\mathrm{BMI}$. There was also a non-significant trend towards a shorter duration of diabetes with increasing deprivation. This suggests that diagnosis could have been delayed in this group. There were no consistent differences by SES cross-sectionally or in changes over time in any of the following: mean levels and attainment of targets for blood pressure and glycaemic control, cholesterol concentrations and BMI; treatment with anti-hypertensives, lipid-lowering drugs and aspirin; and microvascular and macrovascular complications.
The results suggest that either the relation between provision and outcomes of care by SES has been exaggerated in many previous studies or that our findings are atypical because the DIS-supported approach to diabetes care in Salford has ensured that such inequalities have not arisen or been maintained.

Whilst the use of clinically-based data derived during routine clinical care is one of the strengths of this study, it is also perhaps the main potential weakness. Such data are not validated and could be collected using variable and non-standardised methods. However, the data have clinical validity in that it is being used in everyday clinical practice, for example, to assess the adequacy of diabetes and BP control. It will therefore directly inform clinical judgments and treatment decisions. In addition, there is no reason to believe that there was any systematic bias in the measurement of any of the DIS variables which could have produced spurious changes in clinical indicators over time or between different SES quintiles-so the trends and variations shown are not likely to be a result of bias in the data collection process.

The study could potentially be affected by selection bias. This would occur if poorly-controlled patients from deprived areas were less likely to be included on the DIS, or if such patients were less likely to attend follow-up appointments and hence contribute less data than poorly-controlled patients from more affluent areas. The first potential bias is unlikely as all patients with diabetes are entered onto the register and quality control checks are carried out in practices to ensure that this is the case. Also, there are no incentive payments linked to the level of control achieved so no 'perverse incentives' operate to discourage registration with the DIS of poorly controlled lower SES patients.

The second form of selection bias would cause an underestimate of gradients in outcomes and quality of care by SES, if poorly controlled patients from more deprived SES groups attended less than poorly controlled patients from more affluent social groups. However, there is no reason to think that this would occur differentially over time and hence the trends observed should be robust. This is supported by evidence of similar increases in recorded measurements of BP, cholesterol, etc occurring across all SES quintiles during the study period-suggesting that there was no change in the degree of any such bias over time by SES.

Two main messages emerge from these data. Firstly, differences in processes and outcomes of care by SES can be minimised among patients with diabetes. Social deprivation should not be regarded as an insurmountable obstacle to effective care. Improvements in process and outcome measures of diabetes care occurred at a similar rate across the quintiles of deprivation, and by 2000 to 2001 there was little difference in the levels of the physiological variables, prescribing 
of preventive treatments and complication rates by deprivation. Secondly, achieving improvements in BP and lipid concentrations could be a much more realistic goal than glycaemic control. This is supported by data from the UKPDS which showed a gradual deterioration of glycaemic control with time, even in the intensively-treated group [25].

In conclusion, we have been able to show the utility of a comprehensive district-wide clinical dataset to explore SES variations and trends in process of care measures, intermediate outcomes, and complication rates among patients with diabetes in a large urban population with a long-standing district-wide integrated diabetes care programme. We found marked improvements in most, but not all, aspects of diabetes care. There were few variations in the trends of process or outcomes of care by SES. Social deprivation should no longer be regarded as an insurmountable obstacle to the provision of diabetes care.

Acknowledgements. We would like to thank S. Hollis for her helpful comments and advice about the statistical analysis and other aspects of the paper.

\section{References}

1. Chaturvedi N, Stephenson JM, Fuller JH (1996) The relationship between socioeconomic status and diabetes control and complications in the EURODIAB IDDM complications study. Diabetes Care 19:423-430

2. Chaturvedi N, Jarrett J, Shipley MJ et al. (1998) Socioeconomic gradient in morbidity and mortality in people with diabetes: cohort study findings from the Whitehall Study and the WHO Multinational Study of Vascular Disease in Diabetes. BMJ 316:100-105

3. Connolly VM, Kesson CM (1996) Socioeconomic status and clustering of cardiovascular disease risk factors in diabetic patients. Diabetes Care 19:419-422

4. Unwin N, Binns D, Elliott K et al. (1996) The relationships between cardiovascular risk factors and socio-economic status in people with diabetes. Diabet Med 13:72-79

5. Mülhauser I, Overmann H, Bender R et al. (1998) Social status and the quality of care for adult people with Type I (insulin dependent) diabetes mellitus-a population-based study. Diabetologia41:1139-1150

6. Nilsson PM, Johansson S-E, Sundquist J (1997) Low educational status is a risk factor for mortality among diabetic people. Diabet Med 15:213-219

7. Roper NA, Bilous RW, Kelly WF et al. (2001) Excess mortality in a population with diabetes and the impact of material deprivation: longitudinal, population based study. BMJ 322:1389-1393

8. Koskinen SV, Martelin TP, Valkonen T (1996) Socioeconomic differences in mortality among diabetic people in Finland: five year follow up. BMJ 313:975-978
9. Diabetes Control and Complications Trial Research Group (1993) The Effect of intensive treatment of diabetes on the development and progression of long-term complications in insulin-dependent diabetes mellitus. $\mathrm{N}$ Eng $\mathrm{J}$ Med 329:979-985

10. Kelly WF, Mahmood R, Turner S et al. (1994) Geographical mapping of diabetic patients from the deprived inner city show less insulin therapy and more hyperglycaemia. Diabet Med 11:344-348

11. Pringle M, Stewart-Evans C, Coupland C et al.. (1993). Influences on control in diabetes mellitus: patient, doctor, practice, or delivery of care? BMJ 306:630-634

12. Hart JT (1971). The inverse care law. Lancet 1:405-412

13. Leese B, Bosanquet N (1995). Change in general practice and its effects on service provision in areas with different socioeconomic characteristics. BMJ 311:546-550

14. Khunti K, Ganguli S, Lowy A (2001) Inequalities in provision of systematic care for patients with diabetes. Fam Pract 18:27-32

15. Khunti K, Ganguli S, Baker R et al. (2001) Features of primary care associated with variations in process and outcome of care of people with diabetes care. Br J Gen Pract 51:356-360

16. Campbell SM, Hann M, Hacker J et al. (2001) Identifying predictors of high quality care in English general practice: observational study. BMJ 323:784-787

17. North West Public Health Observatory (accessed on 10-09-2002). Information sources; http://www.nwpho.org. uk/information/

18. New JP, Hollis S, Campbell F et al. (2000) Measuring clinical performance and outcomes from diabetes information systems: an observational study. Diabetologia 43:836843

19. Vaughan NJ, Home PD (1995) The UK Diabetes Dataset: a standard for information exchange. Diabetes Audit Working Group of the Research Unit of the Royal College of Physicians. British Diabetic Association. Diabet Med 12:717-722

20. New JP, Burns JA, Hollis S, Bennett J, Young RY (2002) Improving ascertainment rates for macrovascular disease within Electronic Diabetes Records using data linkage. Diabet Med 19 [Supp 2]:218

21. Townsend P, Phillimore P, Beattie A, Helm C (1988) Health and deprivation: inequality and the North. Croom: Helm, London

22. Ramsay L E, Williams B, Johnston GD et al. (2000) British Hypertension Society guidelines for hypertension management 1999: summary. BMJ 319:630-635

23. Diggle PJ, Liang KY, Zeger SL. (1994) Analysis of longitudinal data. Oxford University Press, Oxford

24. UK Prospective Diabetes Study Group (1998). Tight blood pressure control and risk of macrovascular and microvascular complications in type 2 diabetes: (UKPDS 38). BMJ 317:703-713

25. UK Prospective Diabetes Study Group (1998) Intensive blood-glucose control with sulphonylureas or insulin compared with conventional treatment and risks of complications in patients with type 2 diabetes (UKPDS 33). Lancet 352:837-854 